\title{
Effect of aspirin treatment on chondromalacia patellae
}

\author{
GEORGE BENTLEY, IAN J. LESLIE, AND DAVID FISCHER
}

From the University Department of Orthopaedic and Accident Surgery, University of Liverpool, and the Nuffield Department of Orthopaedic Surgery, University of Oxford

SUMMARY Twenty-nine patients ( 21 females and 8 males) with chondromalacia patellae diagnosed by arthroscopy were randomnly allocated to receive aspirin or placebo for 3 months. Clinical and arthroscopic examination after 3 months showed no significant change in symptoms, signs, or macroscopic appearances in either group. Surgical treatment was performed in 14 patients for deteriorating symptoms.

Chondromalacia patellae, softening of the articular cartilage of the patella, is accompanied by fibrillation, fissuring, and erosion of the cartilage. Later, sclerosis of the subchondral bone occurs. The histological appearances of the cartilage are indistinguishable from those of early osteoarthrosis, but a progressive osteoarthrotic process has not been recorded. ${ }^{1}$

Since its first description by Budinger in 1906, ${ }^{2}$ chondromalacia patellae has been of great clinical interest, because diagnosis is often difficult. The chief reason for this is that the aetiology is not known and the correlation between the articular cartilage changes and the clinical systems is poor. ${ }^{3}$

The patients affected by chondromalacia patellae are young, usually from 15 to 35 years old, and many are athletic. Often they are considerably disabled by the symptoms of aching behind the patella, recurrent effusions into the knee, instability of the knee, and crepitus.

Some patients become symptom-free with conservative treatment such as isometric quadriceps exercises, short wave diathermy, avoidance of strenuous activity, immobilisation in a plaster-ofParis cast, and analgesics. However, many have persistent symptoms which make operative treatment necessary. As a result various methods of surgical treatment have been employed including removal of the affected area of cartilage by shaving, ${ }^{4-7}$ shaving of the prominent medial femoral condyle, ${ }^{8}$ transposition of the tibial tubercle, ${ }^{9}$ excision of the affected area of cartilage and drilling of the subchondral bone, ${ }^{10}$ and the more radical procedure of

Accepted for publication 11 February 1980

Correspondence to Professor G. Bentley, University Department of Orthopaedic and Accident Surgery, Royal Liverpool Hospital, Prescot Street, Liverpool L7 8XP. patellectomy. ${ }^{10-14}$ The results of these operative procedures vary from series to series, but most reviews are based on small numbers of patients treated over short periods, so that no method of treatment emerges as superior to the others. Patellectomy appeared to be more certain of relieving symptoms than any other operative procedure in 2 previous reviews. ${ }^{1015}$ However, removal of the patella weakens the quadriceps to some extent in $50 \%$ of the patients and diminishes the likelihood of return to vigorous sporting activity. Also, deterioration of the knee joint with fibrillation of the femoral articular cartilage might be anticipated from prolonged contact of the quadriceps tendon with the femoral groove, although this sequel has not been reported to date in humans.

It would be desirable to develop a method of treating chondromalacia patellae by simple medical means which induced healing of the articular cartilage.

Recent work has revealed that the capacity for repair even in mature articular cartilage may be considerable under certain circumstances. For example, cartilage responds to chemical and physical injuries such as compression, laceration, and exposure to the enzyme papain by increasing production of matrix proteoglycans and collagen. Limited proliferation of chondrocytes also occurs. ${ }^{16-21}$ The mechanism of cartilage breakdown has been clarified, and it is now accepted that matrix degradation is due largely to release of proteolytic cathepsins from intracellular lysosomes which fragment the proteoglycan of the matrix, thus impairing the loadcarrying properties of the articular cartilage. ${ }^{22-24}$ The collagen meshwork loses it support, and the superficial fibres rupture producing fissuring of the articular cartilage surface. This results in pressure on 
neighbouring chondrocytes, leading to further cathepsin release and more matrix breakdown in a vicious cycle. ${ }^{25}$

Simmons and Chrisman ${ }^{26}$ demonstrated that sodium salicylate inhibited the reaction of a cathepsin preparation on cartilage matrix by $20 \%$, thereby allowing synthesis to overtake degradation. In rabbits which had undergone scarification of the articular cartilage of the femoral groove of the knee and had received sodium salicylates in a dose sufficient to maintain a serum level of $15-30 \mathrm{mg} /$ $100 \mathrm{ml},(1 \cdot 09-2 \cdot 17 \mathrm{mmol} / \mathrm{l})$ inhibition of cartilage breakdown was seen. In some instances healing of the scarifications was observed. Using a pressure clamp technique on rabbit knees, Ginsberg et al. ${ }^{27}$ confirmed the results histologically and biochemically. Simmons and Chrisman suggested that salicylates might be valuable in the prevention of articular cartilage breakdown in chondromalacia patellae and other forms of degenerative disease in man.

In a clinical trial involving 10 patients with recurrent subluxation of the patella, administration of aspirin in a dose of $3 \mathrm{~g}$ per day for 2-3 months was associated with diminished fibrillation seen on the under surface of the patella at operation. ${ }^{28}$

In a later paper Chrisman et al. ${ }^{29}$ reported the findings in 39 knees in patients with recurrent dislocation of the patella treated with aspirin in a dose of $3 \mathrm{~g}$ per day for 6-8 weeks. In the control group of 23 knees in 22 patients only 2 showed no evidence of chondromalacia at operation. In the 14 patients (16 knees) treated with aspirin 13 knees showed no chondromalacia. The authors stated that the probability of obtaining this distribution by chance is less than 1 in 10000 . There are 3 criticisms of this study. Firstly, the aspirin was not given on a doubleblind basis, and secondly the state of the patella before administration of aspirin was not known. Thirdly, the serum level of salicylate achieved in each patient was not recorded.

A prospective double-blind clinical study was planned to study the effect of aspirin treatment on the cartilage lesions and the clinical symptoms and signs of chondromalacia patellae in which the patellae were examined before and after treatment with the arthroscope to assess any change which had occurred during treatment.

\section{Patients and methods}

All patients in whom a diagnosis of chondromalacia patellae was made clinically according to the criteria of Robinson and Darracott ${ }^{30}$ were considered for the trial. (These criteria can be summarised as follows. Symptoms: (1) Deep-seated retropatellar ache. (2) Sense of instability when bearing weight. (3) Recurrent effusion of the knee. Physical signs: (1) Pain on direct compression of the patella against the femoral condyle. (2) Pain on resisted extension of the knee. (3) Pain on contracture of the quadriceps against the resistance of the finger placed above the patella.) The following procedure was carried out:

(1) Patellar subluxation was excluded by taking tangential $x$-ray views of both patellae at $30^{\circ}$ and $60^{\circ}$ of flexion. ${ }^{31}$ Anteroposterior and lateral radiographs of both knees in the erect position were routine also.

(2) Initial treatment by conservative means with isometric quadriceps exercises for 10 minutes 4 times a day and analgesics, but excluding aspirin, was carried out.

(3) If the conservative treatment failed over a 3month period, the patients were admitted for arthroscopy to assess the extent of the patellar cartilage involvement.

(4) After the diagnosis had been confirmed at arthroscopy, the lesion was photographed and graded I, II, III, or IV on the classification modified from Outerbridge ${ }^{32}$ and Bentley. ${ }^{15}$ The patientso began to take tablets (Palaprin (aloxiprin) or placebo) dispensed randomnly by the hospital pharmacist. The treatment was continued for a 10-week period and serum salicylate levels were monitored weekly at a constant time approximately 4 hours after ingestion of a dose.

(5) Patients were readmitted after 12 weeks for a repeat arthroscopy and photography of the lesion. The symptoms and signs were recorded on a standard protocol at the time of the first admission, and the examination was repeated at the 3-month readmission. The patients were assessed as 'improved', 'unchanged', or 'worse'. Pre- and posttreatment photographs of the patella were compared 'blind'.

Twenty-two females and 8 males entered this study, a ratio of $2 \cdot 8$ to 1 . The ages ranged from 14 to 38 years, with a mean of 25 years. The right knee was involved in 19 cases and the left in 9 . In 2 patients the symptoms were bilateral, the right knee being worse than the left in one and the left knee worse than the right in the other. The most painful knee was employed for this study. One patient refused to be admitted for rearthroscopy, leaving 29 knees in 29 patients for analysis. Thirteen patients received placebo during the study and 16 received aspirin. Of these, 12 maintained an effective blood level of between 15 and $25 \mathrm{mg} / 100 \mathrm{ml}$ during the 10 -week period and 4 did not, due to side effects or noncooperation. The day-to-day operation of the trial 


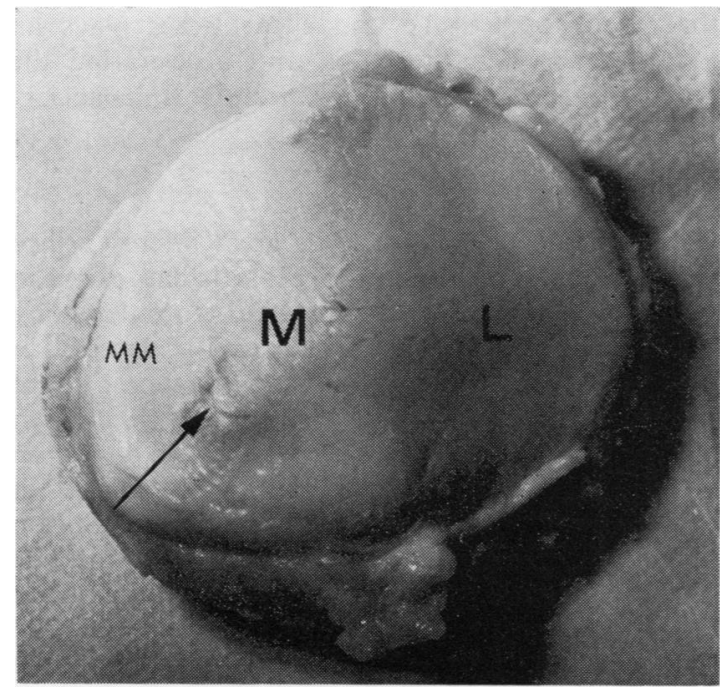

Fig. 1 Posterior surface of right patella showing the 3 facets and an area of chondromalacia (arrow) at the commonest site, namely, ridge at junction of medial and most medial facet. ( $L=$ Lateral facet; $M=$ medial facet; $M M=$ most medial facet.)

was performed by the research registrar, who collected blood samples and advised on side effects and dosage.

\section{LOCATION OF ARTICULAR CARTILAGE CHANGES}

The affected area of the patella was on the junction of the most medial and the main medial facet, or on the medial facet, in 28 of the cases (Fig. 1). In the other case it was on the lateral facet associated with slight lateral subluxation as demonstrated on the tangential $x$-ray views.

\section{Results}

\section{CLINICAL SYMPTOMS AND SIGNS}

Of 10 patients who had improved 6 took effective aspirin throughout the experimental period and 4 took placebo (Table 1). Of 18 who reported no change in symptoms, and in whom there was no change in physical examination, 6 were on effective

Table 1 Clinical results

\begin{tabular}{lllll}
\hline Status & Total & $\begin{array}{l}\text { Effective } \\
\text { aspirin }\end{array}$ & $\begin{array}{l}\text { Noneffective } \\
\text { aspirin }\end{array}$ & Placebo \\
\hline Improved & 10 & 6 & - & 4 \\
Unchanged & 18 & 6 & 3 & 9 \\
Worse & 1 & - & 1 & - \\
\hline
\end{tabular}

aspirin, 3 on noneffective aspirin, and 9 on placebo. One patient who was on noneffective aspirin had worse clinical symptoms and signs. The differences are not significant.

\section{ARTHROSCOPIC APPEARANCES}

Arthroscopy was performed by the senior author in each case by an identical technique, using a lateral approach. After a trial period of comparing arthroscopic appearances with the appearances at arthrotomy, it was possible to estimate the extent of the changes on the under surface of the patella with considerable accuracy. The posterior surface of the patella was first examined and then the remainder of the joint was examined to exclude any other pathology which could have produced the patient's symptoms. Thus the femoral groove and both femoral condyles, the whole of the lateral meniscus, the anterior two-thirds of the medial meniscus, the anterior cruciate ligament, and the infrapatellar fat pad and synovial membrane were examined in every case. Photographs of the posterior surface of the patella were taken through the arthroscope at the initial and the repeat examination. These photographs were then compared on a 'blind' basis.

Table 2 shows the extent of the arthroscopic changes at the beginning and the end of the study. A slight deterioration is obvious overall, with no grade IV changes in the prestudy arthroscopy, but one at the end of the study. There was an increase of 1 in grade III and for grade I a decrease from 7 to 5 patients at the end of the study. The shift in grading is shown in Table 3. In those patients who remained the same or were worse after treatment no significant difference was seen between those who took aspirin and those who took the placebo (Table 4).

Table 2 Arthroscopic grading at beginning and end of study

\begin{tabular}{ccc}
\hline & Pretreatment & Post-treatment \\
\hline Grade I & 7 & 5 \\
II & 13 & 13 \\
III & 9 & 10 \\
IV & - & 1 \\
\hline
\end{tabular}

Table 3 Shift of arthroscopic grading during study

\begin{tabular}{ll}
\hline Grade I & Grade $I \rightarrow$ II $=1$ \\
& Grade I IIII =1 \\
Grade II & Grade I $\rightarrow$ IV $=0$ \\
& Grade III $=0$ \\
Grade III & Grade III $\rightarrow$ IV $=1$ \\
\hline
\end{tabular}


Table 4 Arthroscopic changes during treatment

\begin{tabular}{lrlll}
\hline Status & No. & $\begin{array}{l}\text { Effective } \\
\text { aspirin }\end{array}$ & $\begin{array}{l}\text { Noneffective } \\
\text { aspirin }\end{array}$ & Placebo \\
\hline Improved & 1 & - & - & 1 \\
No change & 25 & 11 & 4 & 10 \\
Worse & 3 & 1 & - & 2 \\
\hline
\end{tabular}

SUBSEQUENT TREATMENT

Some of the patients required surgical treatment for persistent symptoms.

Lateral release of patellar retinaculum $(L R)$. Release of the lateral patellar retinaculum or quadriceps expansion was performed in 4 cases. This procedure, which is experimental at present, appeared empirically to give good results in a proportion of cases who had no lateral subluxation of the patella. It was performed for patients who had a physically demanding job or considerable athletic powers, and in whom there were moderately severe persistent symptoms, with no change in the patellar cartilage appearances at the second arthroscopic examination. In none of these patients were the changes extensive enough to warrant patellectomy.

This procedure appeared to be most useful for grade I or II chondromalacia. One patient with grade III underwent lateral release because of definite evidence of lateral subluxation of the patella, and 1 had cartilage involvement of the lateral facet of the patella. The patient with grade III changes also had a quadriceps lag of $10^{\circ}$, and this was considered to be the least destructive operation which might relieve the pain.

Cartilage excision and drilling of the patella $(C E A D)$. CEAD was performed in 7 patients. This procedure was performed in patients with grade I or grade II changes with worsening symptoms but in whom the changes were not severe enough to warrant patellectomy. The difference between these patients and those who underwent lateral release was that they were more sedentary and had a less demanding occupation or sporting activity.

In 1 patient with grade III changes CEAD was performed because the patient did not have a demanding occupation and the reviewer was not convinced that the symptoms were as severe as stated.

Patellectomy. Three patients underwent patellectomy. They had severe symptoms and signs with retropatellar pain, crepitus in the knee, and effusion with grade III (2) or grade IV (1) changes on arthroscopy. None showed any response to treatment during this study, and patellectomy was the only procedure which would offer relief of symptoms. All of these patients were highly athletic, implying that high compressive and shear stress plays an important part in initiating and accelerating the patellar cartilage breakdown in chondromalacia.

\section{Discussion}

Chondromalacia patellae is of great interest, since it is the only manifestation of articular cartilage breakdown in man which presents before denuding of subchondral bone has occurred. Success in controlling cartilage breakdown at this point might lead to control of more severe cartilage breakdown leading to osteoarthrosis Clinically the condition is a problem because of the high incidence of disabling pain in patients who are young and often athletic. In chondromalacia patellae there is synovial inflammation due to release of articular cartilage matrix proteoglycan and cartilage debris into the synovial fluid. It is probable that aspirin affects the symptoms in these cases by reducing the inflammation of the synovial membrane rather than by any affect on the articular cartilage.

The importance of identifying the condition accurately by arthroscopy and the unreliability of $\overrightarrow{0}$ physical signs was demonstrated in a previous studyo

In this study 10 patients improved, 18 were uns changed, and 1 deteriorated as regards clinicas symptoms and signs. This may be a placebo response.

Table 1 illustrates that Palaprin had no significant effect in reducing symptoms.

The arthroscopic appearances showed a slight general tendency to deteriorate over the 3-month period of the study (Tables 2 and 3). Similarly, improvement was seen (arthroscopically) in only 1 case, and that patient received placebo.

In mature rabbits Chrisman and Simmons ${ }^{26}$ demonstrated healing of lacerations in articular cartilage of the knee when the animals received salicylates in therapeutic dosage. We too could confirm the healing effect of aspirin in lacerated adult rabbit cartilage of the mature femoral head in a proportion of cases $^{\mathbf{3 4}}$ but were unable to demonstrate any such change in chondromalacia patellae. Possibly a trial over a longer period is required, but the great thickness and relative hypocellularity of the patellar articular cartilage over the junction of the medial and most medial facet presumably affects both the nutrition and the capacity to repair damage.

This study was supported by the Arthritis and Rheumatism Council (grant no. B28) for which we are most grateful.

We thank Professor R. B. Duthie for advice and encouragement throughout the study; the consultant and junior staff of the Nuffield Orthopaedic Centre for referring patients for this trial; and the hospital pharmacist, Miss J. Stewart, for making the dispensing arrangements. The Department 
of Biochemistry of the Radcliffe Infirmary at Oxford kindly carried out the estimations of serum salicylate. The Palaprin tablets and placebo were provided by the generosity of Nicholas Laboratories.

\section{References}

1 Karlson S T I G. Chondromalacia patellae. Acta Chir Scand 1940; 83: 347-81.

2 Budinger K. Uber Ablosung von Gelenkteilen und verwandte Prozesse. Dtsch Z Chirurg 1906; 84: 311-265.

3 Leslie I J, Bentley G. Arthroscopy in the diagnosis of chondromalacia patellae. Ann Rheum Dis 1978; 37: 540-7.

4 Chaklin V B. Injuries to the cartilage of the patella and the femoral condyle. J Bone Joint Surg 1939; 21 : 133-40.

5 Cave E F, Rowe C R, Yee L B K. Chondromalacia of the patella. Surg Gynecol Obstet 1945; 81 : 446-50.

6 Wiles P, Andrews P S, Devas M B. Chondromalacia of the patella. J Bone Joint Surg 1956; 38B: 95-113.

7 Wiles P, Andrews P S, Bremner R A. Chondromalacia of the patella. A study of the later results of excision of the articular cartilage. J Bone Joint Surg 1960; 42B: 65-70.

8 Mackenzie Crooks L. Chondromalacia patellae. Early results of a conservative operation. J Bone Joint Surg 1976; 49B: 495-501.

9 Devas M B, Golski A. Treatment of chondromalacia patellae by transposition of the tibial tubercle. $\mathrm{Br}$ Med J 1973 ; i: 588-91.

10 Bentley G. Chondromalacia patellae. J Bone Joint Surg 1970; 52A: 221-32.

11 Soto-Hall R. Traumatic degeneration of the articular cartilage of the patella. J Bone Joint Surg 1945: 27: 426-31.

12 Lewis G B, Holstein A. End result study of patellectomy. J Bone Joint Surg 1958; 40A: 972.

13 Duthie H L, Hutchinson J R. The results of partial and total excision of the patella. J Bone Joint Surg 1958; 40B: 75-81.

14 Evans C D. Chondromalacia patellae. Proc $R$ Soc Med 1966; 59: 626-7.

15 Bentley G. The surgical treatment of chondromalacia patellae. J Bone Joint Surg 1978; 60B: 74-81.

16 Collins D H, McElligott T F, Sulphate (35 S) uptake by chondrocytes in relation to histological changes in osteoarthritic human articular cartilage. Ann Rheum Dis 1960; 19: 318-23.
17 Trias A. Effect of persistent pressure on articular cartilage. An experimental study. J Bone Joint Surg 1961; 43B: 376-86.

18 Meachim G. The effect of scarification on articular cartilage in the rabbit. $J$ Bone Joint Surg 1963; 45B: 150-61.

19 Mankin $\mathrm{H} \mathrm{J}$. The effect of ageing on articular cartilage. Bull N Y Acad Med 1968; 44: 545-52.

20 Repo $R$ V, Mitchell N. Collagen synthesis in mature articular cartilage of the rabbit. J Bone Joint Surg 1971; 53B: 541-8.

21 Bentley G. (1972). Degradation, repair and replacement of articular cartilage. Ch.M. Thesis. University of Sheffield.

22 Fessel J M, Chrisman O D. Enzymatic degradation of chondromucoprotein by cell-free extracts of human cartilage. Arthritis Rheum 1964; 7: 398-405.

23 Ali S Y. The degradation of cartilage matrix by an intracellular protease. $J$ Biochem $1964 ; 93$ : 611-8.

24 Dingle J T. Recent studies on the control of joint damage: the contribution of the Strangeways research laboratory. Ann Rheum Dis 1979; 38: 201-14.

25 Chrisman O D. Biochemical aspects of degenerative joint disease. Clin Orthop 1969; 64: 77-83.

26 Simmons D R, Chrisman O D. Salicylate inhibition of cartilage degeneration. Arthritis Rheum 1965; 8: 960-9.

27 Ginsberg J M, Eyring E J, Lacy S, Tomblin W. Inhibition of cartilage destruction by intermittent salicylate. Arthritis Rheum 1968; 11: 824-9.

28 Chrisman O D, Snook G A. Studies on protective effect of aspirin against degeneration of human articular cartilage. Clin Orthop 1968; 56: 77-86.

29 Chrisman O D, Snook G A, Wilson T C. The protective effect of aspirin against degeneration of human articular cartilage. Clin Orthop 1972; 84: 193-214.

30 Robinson A R, Darracott J. Chondromalacia patellae. Ann Physical Med 1970; 10: 286-90.

31 Ficat P. Les Désequilibres rotuliens de l'hyperpression à l'arthrose. Paris: Masson, 1973.

32 Outerbridge $R$ E. The etiology of chondromalacia patellae. J Bone Joint Surg 1961; 43B: 752-7.

33 Leslie I J, Bentley G. (1975). Arthroscopy in the diagnosis of chondromalacia patellae. Paper presented at the Heberden Society Meeting, London, 20 November, 1976.

34 Forney H J, Bentley G, Mathews R S. Salicylates and repair in adult articular cartilage. Orthopaedics 1973; 6: $19-31$. 